Annales Geophysicae (2002) 20: 289-291 (C) European Geophysical Society 2002

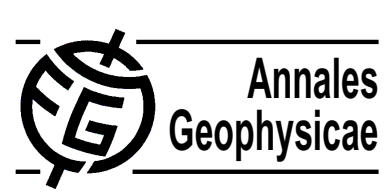

\title{
Introduction
}

\section{The Interball project after 6 years of data analysis}

\author{
L. Zelenyi ${ }^{1}$ and J.-A. Sauvaud ${ }^{2}$ \\ ${ }^{1}$ IKI, Moscow, Russia \\ ${ }^{2}$ CESR, Toulouse, France
}

The Interball project consists of two pairs of satellites orbiting in the Earth's magnetosphere. It was managed by the Russian space agency (RKA) in the framework of a wide international cooperation. The Interball-1 pair, comprising a main Prognoz type satellite and a subsatellite, MAGION4, was launched in August 1996 into a highly elongated (800 km perigee, $190000 \mathrm{~km}$ apogee altitude), $62.8^{\circ}$ inclined orbit which crossed the equatorial parts of the Earth's environment at distances of a few tens of Earth's radii, and was therefore particularly suitable for solar wind/magnetosheath and magnetospheric substorm studies. The main purpose of the subsatellites of the Interball-1 pair was to search for cause-and-effect relationships by performing measurements synchronized with those made on the main satellite. Measurements at two different points in space enable one to distinguish reliably (in one direction, at least) the locations and motions of various plasma structures and/or inhomogeneities, thus making a correct interpretation of experimental results easier.

The Interball-2 pair was launched one year later into a $750 \mathrm{~km}$ perigee, $20000 \mathrm{~km}$ apogee altitude orbit of almost the same inclination, and was devoted to studies of the high altitude auroral and polar cap regions. For an orbit of this type the line of apsides does not rotate, i.e. the apogee stays at constant latitude. The payload of each spacecraft, the Prognoz main satellites and the MAGION-4 and MAGION5 subsatellites included in situ particle and wave measurements.

Interball-1 performed systematic measurements in the solar wind, the magnetosheath, the magnetotail, and over the auroral region, including the middle and high-latitude cusp up to October 2000, when the satellite re-entered into the Earth's atmosphere. Initial results from these spacecraft have previously been published in Annales Geophysicae, Vol. 15, Number 5 (May 1997).

Interball-2 provided a rich collection of data on the high altitude auroral zones at all local times. Its very slow motion near apogee allowed one to study rapid temporal processes, difficult to obtain with a fast orbiting satellite. Initial results from these spacecraft have been published in Annales Geophysicae, Vol. 16, Number 9 (September 1998). Prognoz-type satellites such as Interball-1 and -2, have a so- lar orientation with a slow (120 s) rotation, and normally, the spin axis should be reoriented towards the Sun once every 6 to 8 days. The orbit of the Interball-2, with a low apogee $(\sim 20000 \mathrm{~km})$, was not typical of these spacecraft, since previous Prognoz satellites have been used mainly for the exploration of the outer regions of the magnetosphere and the solar wind. Here, at lower altitudes, the gravity gradient perturbations which accumulated at the perigee segment of the orbit became very significant for the evolution of the orbit and for the stability of the spacecraft orientation. These effects resulted in strong nutation oscillations of the spacecraft (up to $40^{\circ}$ ), even during the early stages of the Interball- 2 mission. A special program for the suppression of this nutation operated automatically every $12 \mathrm{~h}$, and has resulted in a high expenditure of the gas supply of the spacecraft's correcting motors. This unusual situation for a Prognoz-type satellite (the gas resources, for example, on the Interball-1 would allow spacecraft operation well beyond its ballistic lifetime) led to the loss of the Interball-2 orientation capabilities in the autumn of 1998. Data were acquired up to January 1999.

\section{Scientific results}

Both pairs of satellites performed systematic measurements during their lifetime with the Interball-1 coverage reaching $80 \%$. Furthermore, several campaigns were conducted in coordination with the Geotail, Polar and Wind spacecraft. A detailed discussion of the scientific objectives of the mission and its strategy may be found in a paper by Galeev, Galperin and Zelenyi (1996) published in a special issue of Cosmic Research (Vol. 34, (4) p. 310, 1996, a translation of the Russian journal Kosmicheskie Issledovania).

From the beginning of the mission up to today, $\sim 480 \mathrm{pa}-$ pers have been published, mainly in Russian, European and US scientific Journals. Many important results have been published during these years in special issues of Cosmic Research (Vol. 36,(1); Vol. 36 (3); Vol. 36 (6) 1998; Vol. 37 (6), 1999; Vol. 38 (5) 2000; Vol. 40 (4) 2002), Czechoslovak Journal of physics (Vol. 49 (4a) 1999) and in the book "INTERBALL in the ISTP program" edited by D. Sibeck and K. Kudela (Kluwer, 1999). 
This issue of Annales Geophysicae presents 10 more publications devoted to widely different topics. A few papers, having problems in meeting the deadlines, will be published later in a regular issue of the journal.

Three papers published here are devoted to the interplanetary medium and to the direct interaction of the solar plasma with the outer boundary of the magnetosphere.

Dalin et al. compiled and investigated statistically a set of large and sharp solar wind dynamic pressure changes using both Interball-1 and IMP-8 data. The most impressive feature of the large pressure change is their short duration; in about $50 \%$ of the studied events, the change in the dynamic pressure takes less than $1 \mathrm{~min}$. Only $15 \%$ of the events are associated with shocks. The authors rule out the possibility of observing slow mode waves and eliminate the possibility that the events coincide with the interfaces of fast and slow streams. They also show that the events are neither tangential discontinuities, nor compressional waves. A better understanding of the evolution of these structures, possibly related to plasma instability created in the interplanetary medium, is clearly needed.

Safrankova et al. present an extensive study of the magnetopause shape and location, and show that the dayside magnetopause is indented in the cusp region. The deepness of the indentation can reach $\sim 4 R_{E}$ and the dimension of the indention does not depend on the dipole tilt, whereas the location does.

Merka et al. study cusp-like plasma in high-latitudes, mainly from MAGION-4, the sub-satellite of Interball-1. The width of the cusp is an indicator of the strength of the merging process and of the degree of the opening of the magnetosphere. Two prominent results are presented, (i) the major parameter affecting the overall cusp configuration at high-latitudes is the dipole tilt and, (ii) at high altitudes, the cusp occupies a broader region than can be expected from low and middle altitude observations. A comparison with the DMSP, Viking, Polar, and Hawkeye satellite data suggests the widening of the cusp at high altitudes. It is valid for both the latitudinal and longitudinal extensions of the cusp.

The four following papers present different studies of the night-side magnetosphere. Petrukovich and Yermolaev used the fact that the spin axis of the Interball-1 satellite is pointing towards the Sun in order to perform a detailed study of the vertical motion of the magnetotail for different interplanetary magnetic field (IMF) observations. Due to this spin axis orientation, the same angular sector of the CORALL ion spectrometer is successively looking along the $+Z$ and $Z$ axes which allows for an accurate measurement of the small $V z$ plasma velocity. They demonstrate that the plasma convection speed is directed towards the tail mid-plane (equatorial plane), with a mean value of $7 \mathrm{~km} / \mathrm{s}$. This convection velocity vanishes when the $B_{z}$ IMF is directed northward.

Grigorenko et al. present the results of a statistical study of plasma regimes observed in the interface region between the north lobe and the plasma sheet. The data were obtained in the mid-tail at $X$ GSM $=-15$ to $-25 R_{E}$. Different plasma regimes were identified: (1) high energy earthward short- lived ion beams (beamlets), and (2) plasma sheet-like events of various duration. The authors show that for a northward IMF, the characteristic energy of the beamlets decreases with increasing distances to the neutral sheet. A statistical analysis of plasma sheet-like events shows that (i) during northward IMF, short-lived plasma sheet-like "clouds" fill almost all lobes; and (ii) the measured duration of these events is inconsistent with flapping motions of the plasma sheet; hence, they constitute structures detached from the main plasma sheet.

Borodkova et al. present experimental facts from the Interball-1 and Polar satellites showing that in the mid-tail plasma sheet, the plasma flow velocity during substorms is directed tailward when the auroral bulge developed equatorward of the spacecraft's ionospheric footprint. On the contrary, when active auroras moved poleward of the Interball-1 projection, earthward fast plasma flow bursts were observed. These results are interpreted in the framework of the near Earth neutral line model (NENL).

Buzulukova et al. analyze the sharp gradients (decreases) in the fluxes of $\mathrm{H}^{+}$ions seen on board Interball-2 at restricted energy ranges in the inner magnetosphere for a wide MLT range during quiet times. Observations are used to test different global electric field models and plasma source locations in the tail. As a main result, this work demonstrates that despite the existence of large amplitude local fluctuations of the electric field in the inner magnetosphere, a large stationary average convection pattern exists at the root of the observations.

Bouhram et al. present an extensive study of the electrostatic interaction between Interball-2 and the ambient plasma. These authors determine the spacecraft potential from current calculations. As a result, the satellite potential measurements can be used as a diagnostic method to determine the plasma density, and the distribution function of low-energy ions can be corrected from spacecraft charging effects.

Hamelin et al. present computations of low-energy ion trajectories in the complex, 3D electrostatic potential structure built around the Interball-2 spacecraft and use these results to explain some artifacts in the current processing of ion flux data, and to define further improvements of the analysis software.

\section{A key Interball scientist: Yuri Ilyich Galperin}

The paper by Professor Yuri Ilyich Galperin deserves special attention. It deals with the characteristics of the polarization jets and the development of theoretical models. During the preparation of this special issue, at the end of 2001, this key Interball scientist (age 69) suddenly passed away. He was famous for his pioneering works in magnetospheric physics from ground observations and from space missions. Yuri Ilyich was among the initiators and organizers of the Interball project, starting from the creation of an "electromagnetically clean satellite" (including special arrangements for solar pan- 
els and equipotentialization of the satellite surface) and an enthusiastic participation in the elaboration of the Interball scientific program. Yuri was PI and CoI of several experiments aimed at measuring thermal and super thermal plasma on board the Interball-2 satellite (RON, HYPERBOLOID, ANOD) and of the auroral imager UVAI. The main focus of Yuri Galperin during the last few years have been devoted to the measurements of auroral plasma and its modeling in the inner magnetosphere, 3D simulations of electric field distributions near a charged satellite, and the implementation of indirect methods for thermal plasma diagnostic, e.g. for the determination of the plasma density from the charge of the satellite.

\section{Future}

The Interball project completed its first active operational part in October 2000. Scientists and engineers previously involved in the mission control and data processing are now working on the preparation of new projects, which have their roots in the Interball scientific heritage.

One of these missions, ROY (SCHWARM), is currently a Russian-German multi-satellite project, which is aimed at studying multi-scale plasma structures in the "critical" regions of the magnetosphere. The primary targets will be outer cusps, the sub-solar magnetopause, the magnetospheric flanks and the earthward edge of the cross-tail current sheet. Recent findings from Interball and other ISTP spacecraft indicate how important and probably critical is the role of the magnetic field reconnection in these regions. The subsatellites of the ROY project will use GPS technology and will be able to explore plasma processes at scales down to the electron inertia length (tens of $\mathrm{km}$ ), which might reveal new physical effects not found in previous multi-satellite missions.

Another mission extending the capabilities of the Interball system is Interball-3 or Interball-Prognoz, which is under preparation in the Russian and Ukrainian Space Agencies. Interball-3 is thought to be one of the segments of the future international Space Weather Program. A PrognozM1 type satellite will have an orbit with significantly higher apogee $(\sim 400000 \mathrm{~km})$ than that of the Interball-1 spacecraft and will be designed essentially as an upstream monitor. Depending on the type of booster, measurements at the L1 and L2 libration points are also under consideration. A complementary segment of the mission will consist of three

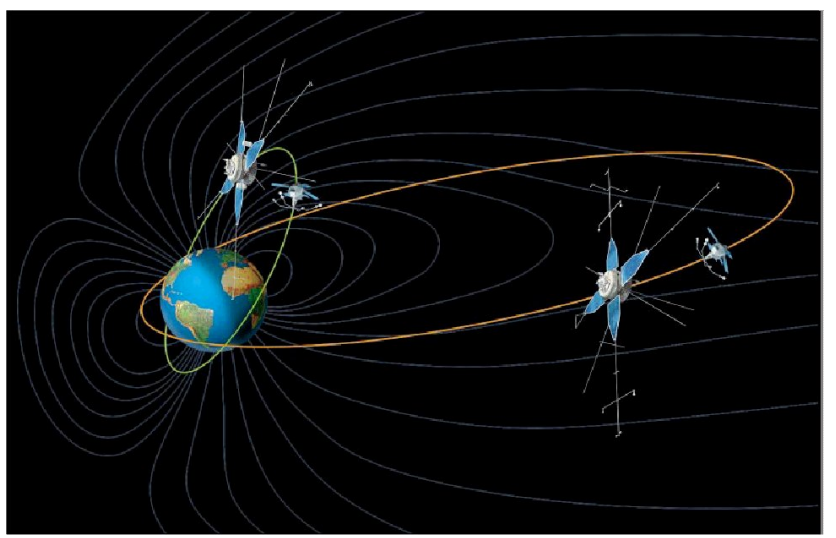

Fig. 1. A schematic view of the Interball mission in the Earth's magnetosphere.

micro-satellites launched into a circular Sun-synchronous orbit oriented along the dawn-dusk meridian with an apogee of $\sim 700 \mathrm{~km}$. The main scientific objective of this mission is the development of a prototype of a Space Weather system, including measurements of solar radiations, solar cosmic rays, IMF and solar wind plasma. At low altitudes, the system will provide continuous real-time data on precipitating particles, field-aligned currents, and cross-polar cap potential. A new micro-satellite is now being designed for this mission by "Youzmash" the Ukrainian space center.

In the framework of the Interball project, a joint experiment with a ground-based powerful HF heating facility has been undertaken to study magnetospheric manifestations of artificial ionospheric heating. Some of the very interesting results of the Interball 1-2 programmes were the measurements of a significant modification of the field-aligned current system and the stimulation of natural magnetospheric disturbances. For a more detailed study of resonant waveparticle interactions in the inner magnetosphere, a successor of the Interball mission, the RESONANCE project, was initiated in 1998. RESONANCE is particularly aimed at studying the dynamics of magnetospheric cyclotron masers, the formation of the ring current and the artificial excitation of wave modes and particle precipitation.

These post-Interball projects are open to international cooperation. The technical and scientific Interball teams will hence continue their successful work in the exploration of the near-Earth space. 